\title{
Particle Filter Tracking of Complex Stochastic Systems Applied to In Silico Wavefront Propagation
}

\author{
Gonzalo R Ríos-Muñoz ${ }^{1,2}$, Antonio Artés-Rodríguez ${ }^{1,2}$, Joaquín Míguez ${ }^{1,2}$ \\ ${ }^{1}$ Signal Theory and Communications Department, Universidad Carlos III de Madrid, Leganés, Spain \\ ${ }^{2}$ Gregorio Marañón Health Research Institute (IiSGM), Madrid, Spain
}

\begin{abstract}
A high dimensional tracking system based on the FithzHugh-Nagumo (FH-N) equations emulating the biological excitation and propagation dynamics of the action potential across cardiac cells is proposed. The modified $F H-N$ model tracks the electric cardiac wavefronts on a tissue, emulating an approximated atrial fibrillation scenario. Bayesian tracking is achieved with two particle filter $(P F)$ schemes: a sequential Auxiliary PF (APF) and a parallelized method, Independent APF (IAPF). The numerical results of the two examples, involving both estimation errors and running times, provide numerical evidence that support the theoretical findings.
\end{abstract}

\section{Introduction}

Atrial fibrillation (AF) is the most common heart rhythm disorder (cardiac arrhythmia), causing substantial morbidity and mortality [1]. However, the precise mechanisms sustaining AF are still not well understood, partly due to difficulties in reliably mapping electrical activity during the spatio-temporal variations of $\mathrm{AF}$ in patients.

The lack of information about the initiation and maintenance of AF has risen the attention of the scientific community, with many theories and assumptions over the past years [2], [3]. However little knowledge about AF is still available if we compare it to other common arrhythmias.

We aim to develop mathematical models to unveil the latent behavior of AF, causes of initiation, macroreentry anchoring mechanisms and possible termination procedures. Bearing in mind these considerations, the chaotic electrical wave propagation in both atria when AF is present makes the developing of complex systems necessary. A particle filter based tracking model with adaptation to external stimuli introducing several unexpected foci is proposed. With the purpose of accomplishing this task a 2dimensional dynamical complex model consisting of a network of modified stochastic FitzHugh-Nagumo (FH-N) oscillators is studied.

\section{Bayesian Tracking}

\subsection{State Space Model}

We selected the FH-N equations by their resemblance to the physiological action potential shape of cardiac cells. We modeled a rectangular grid of $J \times J$ nodes, where each node describes a subsystem following the discretized classical FH-N differential equations using Euler's method with an integration time step $T_{d}$

$$
\begin{array}{r}
U_{i, j, t}=U_{i, j, t-1}+T_{d}\left(\Psi_{i, j, t}+m_{i, j} F_{t}+p_{3}\left(U_{i, j, t-1}\right)\right. \\
\left.-V_{i, j, t-1}+\frac{1}{D} \sum_{(l, r) \in \mathcal{N}_{i, j}} U_{l, r, t-1}\right)+\sigma^{2} \sqrt{T_{d}} B_{i, j, t}, \\
V_{i, j, t}=V_{i, j, t-1}+T_{d}\left(\beta_{0} U_{i, j, t-1}+\beta_{1} V_{i, j, t-1}+\beta_{2}\right),
\end{array}
$$

where the continuous-time stochastic process $U_{i, j, t}$ usually represents an action potential in biological models, and $V_{i, j, t}$ is the so-called recovery process, that evolves according to the differential equation. To enable ectopic foci tracking, we included a stochastic (additive noise) term $\sigma^{2}$, a random stimulus $\Psi_{i, j, t}$, and a coupling term that determines the interaction with the neighbor nodes $D$. A detailed explanation of the rest of the parameters can be found in [4].

We are interested in studying dynamical patterns in the system modeled by the network of Eqs.(1)-(2) caused by the sequence of random stimuli $\Psi_{\mathbf{t}}=\left\{\Psi_{i, j, t}: 1 \leq\right.$ $i \leq j, 1 \leq j \leq J\}_{t \geq 0}$. If a sufficiently strong stimulus is applied to the network in a region that had been recently excited, the propagation of this stimulus can lead to a re-entrant wavefront that can prevail over the excitations caused by the forcing signal $F(s)$.

We propose the following model for the sequence $\boldsymbol{\Psi}_{t}$. For each $t \geq 1$ and a pair of given thresholds $\mathrm{u}_{-}<\mathrm{u}^{+}$, we identify the stimulation region

$$
\begin{array}{r}
\mathfrak{S}_{t}:=\left\{(i, j) \in\{1, \ldots, J\}^{2}: \mathrm{u}_{-}<U_{r, k, t-1}<\mathrm{u}^{+}\right. \\
\text {for some } \left.(r, k) \in\{(i, j)\} \cup \mathcal{N}_{i, j}\right\},
\end{array}
$$


A
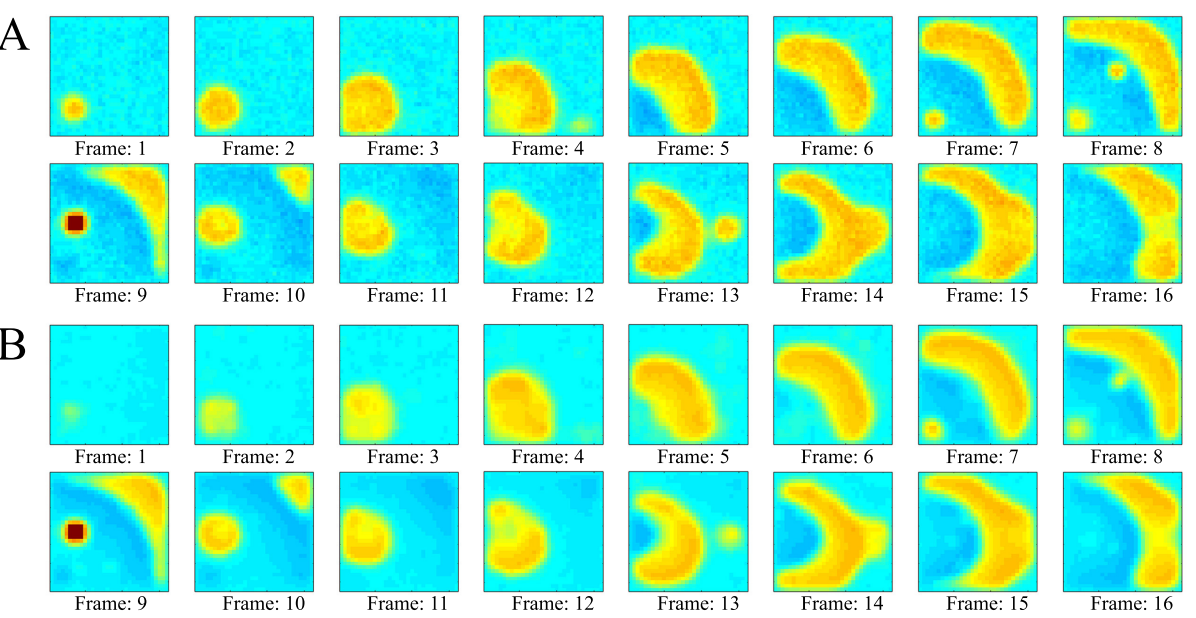

Figure 1. A: Wavefront propagating within a $32 \times 32$ grid, high voltage values displayed in hotter colors. The stimulus is applied in frame 1, and it propagates through the tissue. Random stimuli behind the main wave appear in frames 7-9 and 13. The focal point at frame 9 initiates another wavefront disorganizing the periodic beating scenario. B: Tracking of the IAPF ( $M=10, N=5000)$ for the same simulation. The IAPF detects and tracks the initial stimulus, and it discovers the random stimuli, providing a fine estimation of the evolution of the complex dynamical system.

i.e., $\mathfrak{S}_{t}$ consists of the nodes $(i, j)$ such that the state of the node at time $t-1$ lies between the thresholds, $U_{i, j, t-1} \in\left(\mathrm{u}_{-}, \mathrm{u}^{+}\right)$, or the state of some neighbor at time $t-1,(r, k) \in \mathcal{N}_{i, j}$, lies between the thresholds, $U_{r, k, t-1} \in\left(\mathbf{u}_{-}, \mathbf{u}^{+}\right)$. At each time step, a new stimulus is applied to a group of neighbor nodes in the region $\mathfrak{S}_{t}$ with (a small) probability $\epsilon \geq 0$. To be specific, let

$$
\mathrm{B}_{t}^{\epsilon} \sim \operatorname{Bernoulli}(\epsilon), \quad t=1,2, \ldots,
$$

be a sequence of i.i.d. Bernoulli random variables with $0 \leq \epsilon<1$ then, for each $(i, j) \in\{1, \ldots, J\}^{2}$, and let

$$
\left(i_{t}^{*}, j_{t}^{*}\right) \sim \operatorname{Uniform}\left(\mathfrak{S}_{t}\right)
$$

be a single pair of indices drawn from the uniform distribution with support on the stimulus region $\mathfrak{S}_{t}$. Then, we can generate a random indicator $Q_{i, j, t}^{\epsilon}$ of the form

$$
Q_{i, j, t}^{\epsilon}=\left\{\begin{array}{cl}
0, & \text { if }(i, j) \notin\left\{\left(i_{t}^{*}, j_{t}^{*}\right)\right\} \cup \mathcal{N}_{i_{t}^{*}, j_{t}^{*}} \\
\mathrm{~B}_{t}^{\epsilon} & \text { if }(i, j) \in\left\{\left(i_{t} *, j_{t}^{*}\right)\right\} \cup \mathcal{N}_{i_{t}^{*}, j_{t}^{*}}
\end{array}\right.
$$

which selects a set of neighboring nodes where a new stimulus is to be applied at time $t$. Then, the sequence of stimuli in the $(i, j)$ node can be written as

$$
\Psi_{i, j, t}=\tilde{F} \max \left\{1, \sum_{l=0}^{\ell_{0}-1} Q_{i, j, t-l}^{\epsilon}\right\},
$$

where $\tilde{F}$ is the amplitude of each single stimulus, which is sustained during $\ell_{0}$ consecutive discrete-time steps.

\subsection{Particle Filter}

Concerning the non-linearity of the system equations, the implementation of particle filters [5] based on sequential Monte Carlo (SMC) methodology for estimating the continuously changing propagation wave scenario is adopted. PF algorithms are used as tools for estimating time-varying states of a dynamic system that cannot be observed directly, but through some related measurements. By using a probability mass function (pmf) with random support the approximation of the a posteriori probability distribution of the state given the observations is achieved. This pmf consists of a set of state-space samples and weights pairs, which can intuitively be understood as posterior probabilities.

The unobserved dynamic state is composed of the nodes action potential $\mathbf{U}$, recovery variable $\mathbf{V}$, the candidate stimulus region $\mathfrak{S}$ and the observations, which are the noisy measurements of the observation nodes. A typical particle filter includes three steps that are repeated sequentially:

- Monte Carlo sampling in the space of the state variables,

- computation of weights for the generated samples and,

- resampling according to the weights.

We implemented two algorithms, the Auxiliary Particle Filtering (APF) and the Independent APF (IAPF), using a pool of multi-core CPUs for the implementation of the parallel filters. The details for the algorithms are described in [6]. To characterize the tracking states, let us denote

$$
\begin{aligned}
& X_{i, j, t}=\left(U_{i, j, t}, V_{i, j, t}, Q_{i, j, t-\ell_{0}+1: t}\right) \in \mathbb{R}^{2+\ell_{0}} \text { and } \\
& X_{t}=\left(X_{1,1, t}, \ldots, X_{1, J, t}, \ldots, X_{J, J, t}\right) \in \mathbb{R}^{\left(2+\ell_{0}\right) J^{2}} .
\end{aligned}
$$


The $\left(2+\ell_{0}\right) J^{2}$-dimensional sequence $\left\{X_{t}\right\}_{t \geq 0}$ is a Markov process in discrete time. We do not attempt to write down the associated Markov kernel explicitly, yet it is straightforward to generate a sample $X_{t}$ conditional on $X_{t-1}=x_{t-1}$ using Eqs. (1)-(7), over the set of indices $\{(i, j): 1 \leq i \leq J, 1 \leq j \leq J\}$.

To complete the state-space model description, we assume the ability to observe the signal (voltage) variables, $U_{i, j, t}$, in a subset of the nodes of the grid. To be specific, at time $t$ we collect the measurements

$$
Y_{i, j, t}=U_{i, j, t}+\bar{\sigma}^{2} \bar{B}_{i, j, t}, \quad(i, j) \in \mathcal{S}_{y},
$$

where $\mathcal{S}_{y} \subset\{1, \ldots, J\} \times\{1, \ldots, J\}$ is the set of observed nodes, $\left\{\bar{B}_{i, j, t}:(i, j) \in \mathcal{S}_{y}\right\}$ is a set of i.i.d. standard Gaussian random variables (centered and with unit variance) and $\bar{\sigma}^{2}$ is a known scale parameter. The full observation at time $t$ is denoted

$$
Y_{t}=\left\{Y_{i, j, t}:(i, j) \in \mathcal{S}_{y}\right\} \in \mathbb{R}^{\left|\mathcal{S}_{y}\right|} .
$$

The likelihood function is Gaussian, namely

$$
p\left(y_{t} \mid x_{t}\right) \propto \exp \left\{-\frac{1}{2 \bar{\sigma}^{2}} \sum_{(i, j) \in \mathcal{S}_{y}}\left(y_{i, j, t}-u_{i, j, t}\right)^{2}\right\} .
$$

Equations (3)-(7), (1), (2) and (8) describe a Markov state-space model in discrete time, with conditionally independent observations. We aim at tracking the sequence of probability measures

$\mathbb{P}\left(X_{t} \in A \mid Y_{1: t}=y_{1: t}\right)=\int_{A} p\left(x_{t} \mid y_{1: t}\right) d x_{t}, t=1,2, \ldots, T$,

where $A \in \mathcal{B}(\mathcal{X})$ and $y_{1: T}$ is a given sequence of observations.

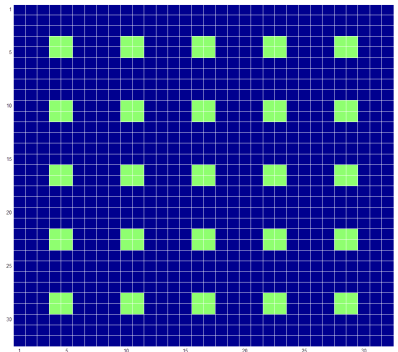

Figure 2. Observation nodes (green) within the $32 \times 32$ grid of stochastic FH-N systems with random stimuli. All nodes follow the dynamics in Eqs. (1)-(7).

\subsection{Simulation setup}

We ran simulations for a network of $1,024(J=32)$ modified stochastic FH-N nodes, interconnected in a regular square grid. We considered as neighbors those nodes contained in a $3 \times 3$ area. The time discretization period was $T_{d}=5 \times 10^{-3}$ continuous-time units and the coupling constant, that sets the "strength" of the links between neighbors, was $\frac{1}{D}=4.5 \times 10^{-3}$.

The dynamics of the FH-N system is highly dependent on the choice of the polynomial $p_{3}(u)$ in Eq. (1), which for this set of simulations is selected as

$$
p_{3}(u)=u\left(u+\sqrt{\frac{18}{5}}\right)\left(u-\sqrt{\frac{18}{5}}\right),
$$

and the forcing signal $F(s)$, which hereafter consists of a periodic sequence of pulses of the form

$$
F(s)=\sum_{k=0}^{\infty} \sqcap\left(s-k S_{\sqcap}\right),
$$

where $\sqcap(s)$ is the square waveform

$$
\sqcap(s)=\left\{\begin{array}{ll}
\tilde{F} & \text { if } 0 \leq s \leq S_{\sqcap} \\
0 & \text { otherwise }
\end{array},\right.
$$

and the period of $F(s)$ is $S_{\sqcap}=20$ time units and the amplitude of the pulses is $\tilde{F}=200$. The discrete-time forcing signal was $F_{t}=F\left(s=t T_{d}\right)$.

To construct the stimulus region $\mathfrak{S}_{t}$ given by Eq. (3) we use the thresholds

$$
\mathrm{u}_{-}=-1.8 \text { and } \mathrm{u}^{+}=-1.6,
$$

which correspond to the back tail of a wave propagating over the network. The parameter of the Bernoulli distribution in (4) is $\epsilon=10^{-3}$, the amplitude of the stimulus in (7) is $\tilde{F}=200$ and it is sustained form $\ell_{0}=25$ discrete time steps. The variance of the noise term in Eq. (1) is $\sigma^{2}=\frac{1}{2}$ and the specification of the dynamics is complete with the parameters $\left(\beta_{0}, \beta_{1}, \beta_{2}\right)=(2.1,-0.6,0.6)$ in Eq. (2).

Since the foci can appear at any space location, some nodes evenly spread over the network grid are used as observations, allowing the model to sense any anomalous perturbation in their neighboring area and propagate the updated information to all the surrounding nodes. The observations are collected at a grid of $5 \times 5$ equally-spaced zones, each zone consisting of four nodes forming a $2 \times 2$ square, as shown in Figure 2. Therefore, we collect observations from $5 \times 5 \times 2 \times 2=100$ nodes out of 1,024 in the network. For each observed node, say in the position $(r, l)$, we obtain the measurement specified by Eq. (8), where the noise variance is $\bar{\sigma}^{2}=\frac{1}{2}$.

\section{Numerical results}

The empirical MSE averaged over 20 independent simulation runs ${ }^{1}$ is displayed in Figure $3 \mathrm{~A}$, which is obtained

\footnotetext{
${ }^{1}$ All simulations were carried out using a multicore Intel( $\left.R\right) \operatorname{Xeon}(R)$ CPU E5-2680v2@2.80GHz under Matlab 32 bits R2012b version with the Parallel Computing Toolbox enabled. 20 different ground truths were calculated and stored so the comparisons between algorithms were fair.
} 

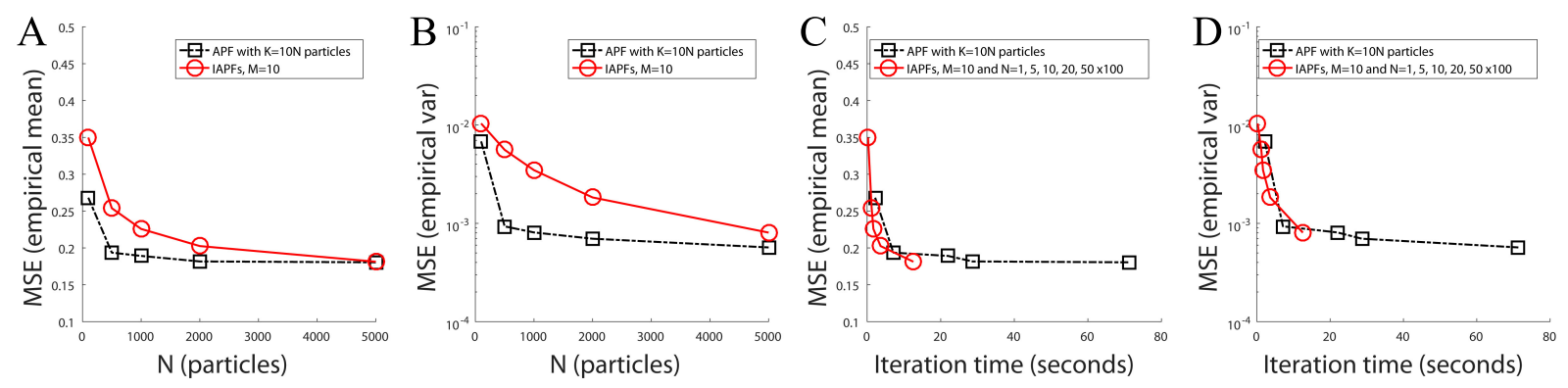

Figure 3. Average tracking results over 20 independent simulation runs, and $N=\{100,500,1000,2000,5000\}$ particles. A: Empirical MSE versus number of particles. B: Variance of the empirical MSE versus the number of particles. C: Empirical MSE versus running time. D: Variance of the empirical MSE versus running time.

by making use of the parallel implementation when the number of particles per filter ranges from from $N=100$ to $N=5000$ and the number of filters is fixed to $M=10$. Then, the total number of particles $K=M N$ associated to the centralized APF ranges from $K=10 \times 100$ to $K=10 \times 5000$, is also shown for comparison. The IAPF achieves worse performance for fewer particles per filter $N$, around $N=100$, while for modest values $N \geq 2000$ ) it nearly matches the MSE of the centralized BF. The empirical variance of the MSE for the same set of 20 simulation trials is displayed in Figure 3B. The results show, that the proposed independent bootstrap filter falls short of the APF as $N$ becomes larger following the same tendency.

Lastly, the running time against the MSE values is examined. Fixing the number of filters to $M=10$, for each value of particles per filter $N=100,500,1000,2000$ and 5000, 20 different independent simulations of the FH-N 2D grid have been run calculating their empirical MSE and the average running time for the parallel IAPF for each combination of $M$ and $N$. Subsequently, the APF was run with $K=M N$ particles, hence for $K=1 \times 10^{3}, 5 \times$ $10^{3}, 10 \times 10^{3}, 20 \times 10^{3}$ and $50 \times 10^{3}$.

Empirical MSE results versus the running time for the two methods are displayed in Figure 3C-D. We define one algorithm as more efficient than the other when it is able to achieve lower MSE in the same amount of time. Then, taking a look at Figure 3C, we can easily observe that the ensemble of $M=10$ IAPF with $N=5000$ particles per filter achieves an empirical MSE of $\approx 0.18$ with a running time of $\approx 12.56$ seconds, while the centralized APF attains the same performance with $K=10 \times 2000$ particles and a running time of $\approx 28.67$ seconds. Therefore same MSE but greatly reduced running time for tracking the system.

\section{Conclusions and Future Lines}

We were able to track random ectopic activity in an interconnected network resembling atrial tissue. We used Bayesian filtering algorithms and took advantage of new parallelized methods for speeding up computation times. Future work will involve the implementation of realistic action potential models, and employing real electrograms from multi-electrode catheters to perform tracking of electrical activity.

\section{Acknowledgements}

This work has been partly supported by MINECO/FEDER (ADVENTURE, id. TEC2015-69868-C2-1-R), and Comunidad de Madrid (project CASI-CAM-CM, id. S2013/ICE-2845).

\section{References}

[1] Kirchhof P, et al. 2016 ESC Guidelines for the management of atrial fibrillation developed in collaboration with EACTS. Europace 2016;18(11):1609-1678.

[2] Jaïs P, et al. A focal source of atrial fibrillation treated by discrete radiofrequency ablation. Circulation 1997;95(3):5726.

[3] Haïssaguerre M, et al. Spontaneous initiation of atrial fibrillation by ectopic beats originating in the pulmonary veins. $\mathrm{N}$ Engl J Med 1998;339(10):659-666.

[4] Luengo D, Ríos-Muñoz G, Elvira V, Sánchez C, ArtésRodríguez A. Hierarchical algorithms for causality retrieval in atrial fibrillation intracavitary electrograms. IEEE Journal of Biomedical and Health Informatics 2018;1-1.

[5] Djurić PM, et al. Particle filtering. IEEE Signal Processing Magazine September 2003;20(5):19-38.

[6] Crisan D, Míguez J, Ríos-Muñoz G. On the performance of parallelisation schemes for particle filtering. EURASIP Journal on Advances in Signal Processing 2018;2018(1):31.

Address for correspondence:

Gonzalo R. Ríos-Muñoz

Universidad Carlos III de Madrid

Department of Signal Theory and Communications Lab. 4.3.B01

Avda. de la Universidad, 3028911 Leganés (Madrid), SPAIN griosm@tsc.uc3m.es 